\title{
REV3L Gene Variants rs1002481, rs462779, and rs465646 Lead to Increased Susceptibility Towards Non-Small Cell Lung Cancer in the Population of Jammu and Kashmir.
}

\section{Rajeshwar Singh Jamwal}

Shri Mata Vaishno Devi University

Nikita Mahajan

Shri Mata Vaishno Devi University

Gh. Rasool Bhat

Shri Mata Vaishno Devi University

Amrita Bhat

Shri Mata Vaishno Devi University

Bhanu Sharma

Shri Mata Vaishno Devi University

Ruchi Shah

University of Kashmir

Minerva Sharma

Shri Mata Vaishno Devi University

\section{Sonali Verma}

Shri Mata Vaishno Devi University

\section{Divya Bakshi}

Shri Mata Vaishno Devi University

\section{Rahul Sharma}

Government Medical College Jammu

\section{Deepak Abrol}

Government Medical College Kathua

\section{Rakesh Kumar}

Shri Mata Vaishno Devi University

Audesh Bhat ( $\sim$ audeshs2002@gmail.com )

Central University of Jammu https://orcid.org/0000-0002-2892-3910

Research article 
Keywords: REV3L (Protein reversion less 3-like), Non-small cell lung cancer (NSCLC), DNA Repair Gene, DNA polymerase zeta

Posted Date: July 22nd, 2020

DOI: https://doi.org/10.21203/rs.3.rs-42137/v1

License: (c) (1) This work is licensed under a Creative Commons Attribution 4.0 International License. Read Full License 


\section{Abstract}

Background: Non-small cell lung cancer (NSCLC) is the most prevalent type of lung cancer accounting for $80-85 \%$ of all lung cancer cases. Various genetic studies have tried to reveal the association of $R E V 3 L$ (Protein reversion less 3-like) gene mutations with cancer, including lung cancer but no such study has been carried out in the population of Jammu and Kashmir (J\&K).

Methods: The selected REV3L variants were genotyped using the TaqMan allele discrimination assay in 550 subjects ( 203 NSCLC patients and 347 healthy controls). The association of variants was evaluated by logistic regression.

Results: Out of the four REV3L variants genotyped, we found rs 1002481, rs 462779 , and rs465646 significantly associated with NSCLC risk with an Odds Ratio (OR) of 3.5 $(1.98-6.3$ at $95 \% \mathrm{Cl}), p$ value $=0.00002 ; \mathrm{OR}=4.4(1.8-10.4$ at $95 \% \mathrm{Cl}), p$ value $=$ $0.00075 ;$ and $\mathrm{OR}=2.4(1.47-4.008$ at $95 \% \mathrm{Cl}), p$ value $=0.00053$, respectively . Conclusion: Our data suggest a strong association of variants rs 1002481 , rs 462779, rs 465646 with NSCLC. This association is indicative of a potential role of mutations in the REV3L gene as risk factor for the development of NSCLC in the studied population. Although a first report from any Indian population, these variants have been previously reported to be associated with lung and colorectal cancers in different world populations. These data along with our data supports the notation that these variants can be used as potential prognostic biomarker.

\section{Background}

The region of Jammu and Kashmir (J\&K) in India has long been considered as an endemic cancer zone with a peculiar cancer profile (Rasool et al, 2012) [1]. Lung cancer, which globally accounted for $18.4 \%$ of cancer related deaths in 2018, is a major cancer type in Indian populations, especially in the male population (India State-Level Disease Burden Initiative Cancer Collaborators, 2016; Ferlay et al. 2018)[2]. 
One recent study has put lung cancer as the second most common cancer in the J\&K region with $23.5 \%$ of all new cases in 2017 [3]. Although several studies have evaluated mutations in key genes, such as genes involved in cell cycle and cell growth regulation, in DNA damage repair pathways and many other pathways with reference to different cancer types in the population of J\&K [4-7], none has evaluated the role of Translesion Synthesis (TLS) polymerase in any cancer type. The DNA sequence can get altered as the result of replication error and by the environmental agents such as tobacco smoking, mutagenic chemicals, and certain types of radiation, which may lead to cancer[8-10]. However, various DNA repair pathways, including DNA damage tolerance (DDT) pathway are responsible for maintaining the genomic stability. Any disruption in these pathways, therefore, has a potential to cause malignancy. TLS polymerases, the key players of the DDT pathway consist of a series of specialized polymerases such as polymerases $\mathrm{K}, \zeta, \eta, \mathrm{\iota}$ and Rev1[11-13]. Due to their error prone nature, most of these TLS polymerases, including pol $\zeta$ have been found associated with different cancer types[14]. DNA pol $\zeta$ is a heterodimer, consisting of Rev3, the catalytic subunit and Rev7, the accessory subunit.

Pol $\zeta$ can mediate DNA replication bypassing DNA damage, which may prevent chromosome instability in cells and be considered as a suppressor of spontaneous tumorigenesis at the replication forks thus helping in maintaining the genomic stability, However, several point mutations in $R E V 3 L$ cause proteins misfolding and mis localization, which in turn alters their interactions and biological functions. These dislocations are usually caused by genetic mutations that cause fold level expressional changes and alter protein functions. Therefore, any reduction in the activity of pol $\zeta$ makes cells, including tumour cells sensitive to the DNA-damaging agents and its absence can also cause increased chromosome rearrangements and inflammation thus, promoting carcinogenesis[14-17].

In the present study, we conducted an association study of rs1002481 (T > A), rs462779 (G > A), rs465646

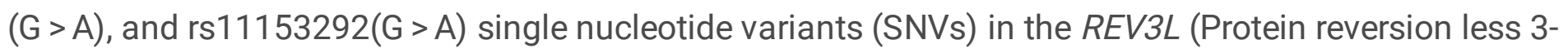
like) gene located on chromosome 6q21 (Chr 6: 111,299,028-111,483,71) with NSCLC in the population of Jammu and Kashmir. These variants were selected based on literature survey as they play an important role in carcinogenesis. SNV rs1002481 is an intronic variant located on Intron 7, rs462779 is an exonic variant located on exon 15, rs465646 is located on 3' UTR region of REV3L and rs11153292 is an intronic variant located on Intron 6.

\section{Methods}

\section{Ethical Statement}

The study was approved by the Institutional Ethics Review board (IERB) of Shri Mata Vaishno Devi University (SMVDU) vide IERB Serial No: SMVDU/IERB/16/41. The informed written consent was taken into account from each participant and all the parameters were recorded in pre-designed Performa. In this study all experimental research work was performed according to guidelines issued by Institutional Ethics Review board committee.

\section{Sampling}


A total of 550 subjects, (203 NSCLC cases and 347 healthy controls) were recruited for the study. All cases were histopathological confirmed. The genomic DNA was isolated from the blood samples using the FlexiGene ${ }^{\circledR}$, Qiagen DNA Isolation Kit (catalogue No. 51206). Agarose gel electrophoresis was used to analyse the quality of genomic DNA and quantification was performed using Eppendorf BioSpectrometer ${ }^{\circledR}$ (Nanodrop).

\section{Genotyping}

Genotyping of the selected SNVs was performed using the allele discrimination assay on the MX3005p Agilent Real-time PCR platform and we adopted the methodology from our previous studies $[4,6]$. TaqMan Probes rs1002481(VIC:A \& FAM:T) rs462779(VIC:A \& FAM:G), rs465646(VIC:A \& FAM:G), and rs11153292(VIC:A \& FAM:G) labelled with VIC and FAM (Thermofisher Scientific) and UNG Master Mix (Applied Biosystems, USA) were used for genotyping. Dilutions of the assay were made from 40X concentration to 20X using TE (Tris EDTA buffer) as per the manufacturers protocol. The volume of the total PCR reaction was $10 \mu \mathrm{l}$, comprising of $2.5 \mu \mathrm{l}$ of TaqMan UNG Master Mix, $0.25 \mu \mathrm{l}$ of the probe, $3 \mu \mathrm{l}$ DNA $(5 \mathrm{ng} / \mu \mathrm{l})$ and $4.25 \mu \mathrm{l}$ nuclease-free water to make up the final volume. The following thermal conditions were adopted; hold for $10 \mathrm{~min}$ at $95^{\circ} \mathrm{C}$, then 40 cycles of $95^{\circ} \mathrm{C}$ for $15 \mathrm{~s}$ and $60^{\circ} \mathrm{C}$ for $1 \mathrm{~min}$. All the samples were run in 96-well plates with three no template control (NTC). The post PCR detection system was used to measure allele-specific fluorescence. Ninety-three random samples were picked and re-genotyped for cross-validation of genotyping calls and the concordance rate was $100 \%$.

\section{Statistical analysis}

Genotypic frequencies of each variant were tested for Hardy-Weinberg equilibrium by performing Chisquare test (X2) and then the variants that followed HWE were further analyzed to check there association with NSCLC by using IBM SPSS software v.23.0 and the associations between REV3L variants and nonsmall cell lung cancer risk were estimated by calculating the ORs at $95 \% \mathrm{Cl}$ by Binary logistic regression analyses with adjustment for Age, Gender, Body Mass Index, Alcohol consumption, and smoking status and we also calculated the statistical power of each variant by using PS software.

\section{Results}

We recruited a total of 550 subjects, out of which 203 were NSCLC patients (cases) and 347 healthy individuals (controls). Among the cases, $82 \%$ were males and $18 \%$ were females and among the controls, $75 \%$ were males and $25 \%$ were females, suggesting that the prevalence of NSCLC is high among males in the $\mathrm{J} \& \mathrm{~K}$ population. The mean age of cases and controls was $61.38 \pm 9.7$ years and $41.68 \pm 16$ years, respectively which were slightly statistically different ( $p$-value $=6.36 \mathrm{E}-29)$. The average $\mathrm{BMI}$ of cases was $22.13 \pm 3.75 \mathrm{~kg} / \mathrm{m}^{2}$ and of controls was $23.01 \pm 5.2 \mathrm{~kg} / \mathrm{m}^{2}$ ( $p$-value=0.034). The distribution of other clinical characteristics between cases and controls are given in table 1 .

\section{Table 1: Clinical Characteristics of cases and controls.}




\begin{tabular}{|llll|}
\hline Characteristics & Cases $(\mathbf{n}=\mathbf{2 0 3})$ & Controls $(\mathrm{n}=\mathbf{3 4 7})$ & p-value \\
\hline 1.Age (Years) & $61.38 \pm 9.7$ & $41.68 \pm 16$ & $6.36 \mathrm{E}-29$ \\
\hline 2.Gender & Male: $-82 \%$ & Male: $-75 \%$ & \\
& Female: $-18 \%$ & Female: $-25 \%$ & \\
\hline 3.BMI* & $22.13 \pm 3.75$ & $23.01 \pm 5.2$ & 0.034 \\
\hline 4.Histological Subtypes** & AC: $-65 \%$ & - & \\
& SCC: $-32 \%$ & - & \\
& UDC: $-3 \%$ & - & \\
\hline 5.Smoking & $74 \%$ & $24 \%$ & \\
Yes & $26 \%$ & $76 \%$ & \\
No & & & \\
\hline 6.Alcohol & $34 \%$ & $16 \%$ & \\
Yes & $66 \%$ & $84 \%$ & \\
No & & & \\
\hline
\end{tabular}

*Body Mass index (in $\mathrm{kg} / \mathrm{m}^{2}$ ), **(AC: Adenocarcinoma; SCC: Squamous cell carcinoma; UDC: Undifferentiated carcinoma).

Out of the four SNVs genotyped, three were found significantly associated with NSCLC with odds ratio $(\mathrm{OR})=1.81(1.42-2.32$ at $95 \% \mathrm{Cl})(p$ value $=0.0000021)$ for $\mathrm{rs} 1002482, \mathrm{OR}=1.47(1.13-1.92$ at $95 \% \mathrm{Cl})(p$ value $=0.0039)$ for rs 462779 and $\mathrm{OR}=1.64(1.26-2.14$ at $95 \% \mathrm{Cl}$ ) ( $p$ value $=0.00023)$ for rs 465646 (Table 2). The distribution of risk allele is shown in figure 1 . The differences in the allelic frequency distribution of rs11153292 variant between cases and controls was statistically insignificant (hence data not presented in this manuscript).

Table 2: Allelic Frequency distribution and risk associated with the REV3L variation in Jammu and Kashmir Population 


\begin{tabular}{|lllllll|}
\hline SNP ID & $\begin{array}{l}\text { Cases } \\
(\mathbf{n}=203)\end{array}$ & $\begin{array}{l}\text { Controls } \\
(\mathrm{n}=347)\end{array}$ & HWE* & RA & AllelicOR & $p$-value \\
\hline rs1002481 & $\mathrm{A}-0.57$ & $\mathrm{~A}-0.42$ & 0.76 & $\mathrm{~A}$ & $1.81(1.42-2.32)$ & 0.0000021 \\
& $\mathrm{~T}-0.43$ & $\mathrm{~T}-0.58$ & & & & \\
rs462779 & $\mathrm{A}-0.35$ & $\mathrm{~A}-0.27$ & 0.59 & $\mathrm{~A}$ & $1.47(1.13-1.92)$ & 0.0039 \\
& $\mathrm{G}-0.65$ & $\mathrm{G}-0.73$ & & & & \\
\hline rs465646 & $\mathrm{A}-0.72$ & $\mathrm{~A}-0.61$ & 0.062 & $\mathrm{~A}$ & $1.64(1.26-2.14)$ & 0.00023 \\
& $\mathrm{G}-0.28$ & $\mathrm{G}-0.39$ & & & & \\
\hline
\end{tabular}

Figure 1: Risk Allele frequency in the populations of Jammu and Kashmir (Datawrapper).

In order to explore the maximum effect of the risk alleles, we evaluated the association using the binary logistic regression analysis of different genetic models and observed that under recessive model, SNVs rs 1002481, rs462779, rs465646 were found to be significantly associated with NSCLC risk with adjusted $\mathrm{OR}^{\star}$ values of $3.5(1.98-6.3$ at $95 \% \mathrm{Cl}$ adjusted); $p$ value $=0.00002,4.4(1.8-10.4$ at $95 \% \mathrm{Cl}$ adjusted); $p$ value $=0.00075$, and $2.4(1.47-4.008$ at $95 \% \mathrm{Cl}$ adjusted); $p$ value $=0.00053$, respectively (Table 3 ).

Table 3: Allelic Frequency distribution and risk associated with the REV3L variation under different genetic models in Jammu and Kashmir Population

\begin{tabular}{|c|c|c|c|c|c|}
\hline SNP ID & $\mathrm{RA}$ & Genetic Model & Genotype & p-value & OR*(at $95 \% \mathrm{Cl})$ \\
\hline \multirow[t]{3}{*}{ rs1002481 } & \multirow[t]{3}{*}{$A$} & Dominant model & AA / AT vs TT & 0.0044 & $2.23(1.28-3.8)$ \\
\hline & & Recessive Model & AA vs AT/TT & 0.00002 & $3.5(1.98-6.3)$ \\
\hline & & Additive Model & - & 0.000021 & $2.123(1.5-3.004)$ \\
\hline \multirow{3}{*}{ rs462779 } & \multirow[t]{3}{*}{$A$} & Dominant model & $\mathrm{AA} / \mathrm{AG}$ vs $\mathrm{GG}$ & 0.6247 & $1.12(0.695-1.8)$ \\
\hline & & Recessive Model & $A A$ vs $A G / G G$ & 0.00075 & $4.4(1.8-10.4)$ \\
\hline & & Additive Model & - & 0.054 & $1.4(0.99-2.07)$ \\
\hline \multirow[t]{3}{*}{ rs465646 } & \multirow[t]{3}{*}{$A$} & Dominant model & $\mathrm{AA} / \mathrm{AG}$ vs $\mathrm{GG}$ & 0.0133 & $2.6(1.2-5.7)$ \\
\hline & & Recessive Model & $A A$ vs $A G / G G$ & 0.00053 & $2.4(1.47-4.008)$ \\
\hline & & Additive Model & - & 0.0034 & $1.9(1.35-2.8)$ \\
\hline
\end{tabular}

*OR adjusted for age, gender, $\mathrm{BMI}$, alcohol, and smoking and $p$-values $<0.05$ was considered significant.

\section{Discussion}


Since the advent of genome-wide association studies (GWAS), rapid progress has been made in the identification of lung cancer susceptibility genes, such as those on chromosomes $5 p 15.33,6 p 21$, and 15q24-25.1 [18]. Besides this, individual gene mutation profiling studies and the case-control association studies have also contributed significantly in the identification of lung cancer susceptibility genes such as EGFR, TP53, and RB1 [18, 19]. TLS polymerases, although play an important role in preventing more deleterious effect of stalled replication fork their error prone nature often result in the introduction of genomic instability, a potential cause for malignancy [14]. The role of Rev3 in the maintenance of common fragile sites, further reinforces the role of TLS polymerases in cancer[20]. Consistent with their potential role in cancer, several studies have investigated the association of SNVs in these genes with different cancers including lung cancer[21-23]. However, the association of TLS gene SNVs with any cancer in the population of $J \& \mathrm{~K}$ has not yet been studied. This is a first study analyzing the role of $R E V 3 L$ genetic polymorphisms in NSCLC in the population of J\&K. We looked into the association of three SNVs with NSCLC to understand the role of REV3L germline polymorphisms in increasing the susceptibility towards NSCLC in the studied population. A statistically significant association with NSCLC was observed for genetic variants rs 1002481 , rs 462779 , and rs465646 with adjusted odds ratio (OR*) of 3.5 ( 1.98-6.3 at $95 \% \mathrm{Cl}$ adjusted), 4.4 (1.8-10.4 at 95\% $\mathrm{Cl}$ adjusted), 2.4 (1.47-4.008 at $95 \% \mathrm{Cl}$ adjusted), respectively suggesting that $R E V 3 L$ polymorphisms may have a role in the pathological process of NSCLC in the studied population.

By using Insilco tool SNIPA, we found that genetic variants rs 1002481 and rs465646 play a direct regulatory role and rs 462779 has a direct effect on the transcript. All the three SNPs were found to be in Linkage disequilibrium ( $L D r^{2} \geq 0.8$ ). The plot symbol of each variant indicates its functional annotation (Figure 2).

\section{Figure 2: Linkage disequilibrium plot and the plot symbol of each variant indicating their functional annotation[24].}

The mechanism of pol $\zeta$ mediated lesion bypass involves the binding of Rev 1 to the replication complex which then recruits DNA pol $\zeta$ to the damaged site. Rev3 does not bind directly to Rev1, instead it binds to $M A D 2 L 2$, the accessory subunit of pol $\zeta$ during the formation of the pol $\zeta$ complex and this leads to conformational change in the $\mathrm{C}$-terminal domain of MAD2L2 that binds with the C-terminus of Rev1 to carry out the DNA translesion synthesis [14]. The lesion bypass allows the replication process to continue, thereby preventing the more deleterious effect of blocked replication forks which may lead to chromosome instability in cells (Supplementary figure S1). Hence pol $\zeta$ along with other TLS polymerases may be considered as suppressors of spontaneous tumorigenesis at the replication forks thus helping in maintaining the genomic stability $[16,17]$. REV $3 L$ is shown to interact with many genes as visualized in String tool software v 10.5[25] (Supplementary figure S2) .

Any molecular alteration in the REV $3 L$ gene can cause misfolding and mis localization of Rev3 thus leading to weakened interaction between Rev3 and MAD2L2 (also known as Rev7)[26]. As a result, this will cause under-expression of pol $\zeta$ complex and therefore a compromised lesion bypass [27]. On the 
other hand, nucleotide changes such as rs 465646 in the 3'UTR region of REV $3 L$, a microRNAs binding site can cause over-expression of $R E V 3 L$ which again will lead to compromised lesion bypass mechanism. Hence, both over-expression and under-expression of TLS polymerases can increase the rate of mutation, signifying that they could act equally as oncogenes and tumour suppressor genes as shown in figure 3 [28].

Figure: 3 Schematic representation of molecular alteration in REV3L leading to both over-expression and under-expression of DNA polymerases Zeta that can increase the rate of mutation thus, promoting carcinogenesis[27].

\section{Conclusion}

The statistically significant association of rs1002481, rs462779, and rs465646 with non-small cell lung cancer, which are in linkage-disequilibrium suggests an increased genetic risk factor in the population of J\&K for the non-small cell lung cancer. The association of TLS polymerases with the development of chemoresistance adds one more dimension in the importance of evaluating these polymerases in the cancer patients of this cancer prone geographic region. In the modern era of medical sciences, the discovery of an effective treatment for cancer, including lung cancer remains a daunting challenge due to increase in drug resistance. Therefore, by identifying and targeting the chemoresistance causing genes can prove critical milestone in improving prognosis. The importance of this study, therefore, lies in the identification of nucleotide changes in the TLS polymerases in cancer patients which might prove to be a prognostic or predictive biomarker in the study population.

\section{Abbreviations}

$\mathrm{BMI}$

Body Mass Index; AC:Adenocarcinoma; SCC:Squamous cell carcinoma; UDC Undifferentiated carcinoma; OR

Odds Ratio; Cl:Confidence Interval; HWE:Hardy

Weinberg equilibrium; NSCLC

Non-Small Cell Lung Cancer; SNV:Single Nucleotide

Variant; J\&K

Jammu and Kashmir.

\section{Declarations}

\section{Acknowledgement.}

All authors acknowledge all the participants for their contribution in this study and R.K and R.J acknowledge Indian Council of Medical Research (5/10/15/CAR-SMVDU/2018-RBMCH) and Department of Science and Technology (DST/SSTP/ J\&K/459), Govt. of India for financial assistance, R.J 
acknowledges Dr. Varun Sharma for assisting in Data Analysis. A.B acknowledge the financial assistantship from University Grants Commission, UGC-BSR Start-up Grant (grant no. f.30-352/2017) for purchase of probes.

\section{Authors Contributions}

R.K, A.B, and R.J planned the work. R.J, N.M, B.S and M.S performed experimental work in lab. G.R, A.B, R.S, S.V and D.B helped in sample collection. R.J performed the statistical analysis, wrote the manuscript and drafted the manuscript according to journal. D.A and R.S provided samples for the study. A.B, R.K and R.S finally refined the manuscript. All authors finally revised and approved the manuscript.

\section{Funding}

No Funding.

\section{Availability of data and materials}

Data generated and analyzed during study is not available publicly but can be made available from the corresponding author upon reasonable request.

\section{Ethics approval and consent for participation}

The study was approved by the Institutional Ethics Review board (IERB) of Shri Mata Vaishno Devi University (SMVDU) vide IERB Serial No: SMVDU/IERB/16/41. The informed written consent was taken into account from each participant and all the parameters were recorded in pre-designed Performa. In this study all experimental research work was performed according to guidelines issued by Institutional Ethics Review board committee.

\section{Consent for publication.}

Our manuscript does not contain any individual person's data in any form (including any individual personal details, images or videos).

\section{Corresponding authors}

Correspondence to Dr. Audesh Bhat and Dr. Rakesh Kumar

\section{Competing interest}

The authors declare no competing interest.

\section{Author details}

M.sc Students, School of Biotechnology, Shri Mata Vaishno Devi University, Katra, India. Email: rsjamwal0@gmail.com,mahajannikita22@gmail.com. 
Rajeshwar Singh Jamwal, Nikita Mahajan

Research Scholar, School of Biotechnology, Shri Mata Vaishno Devi University, Katra, India. Email: seithbhat11@gmail.com, amritabhatt88@gmail.com, bhanugaur91@gmail.com, Sharmamanii1322@gmail.com and divya.bakshi09@gmail.com.

Gh. Rasool Bhat, Amrita Bhat, Bhanu Sharma, Minerva Sharma, Divya Bakshi

Department of Biotechnology, Kashmir University, Jammu \& Kashmir, India, Email:

scientistdobt@gmail.com

Ms. Ruchi Shah Scientist 'B'

Indian Council of Medical Research-Centre for Advance Research, Shri Mata Vaishno Devi University Katra J\&K, India, Email: Sonali.verma@smvdu.ac.in

Dr. Sonali Verma, Scientist 'B'

Associate Professor Department of Radiotherapy, Govt. Medical College Jammu. Email: drrahulsharma2002@yahoo.com.

Dr. Rahul Sharma

Assistant Professor, Radiation oncology Govt. Medical College Kathua. Email: deepakabrol2003@gmail.com

Dr. Deepak Abrol

Assistant Professor, Centre for Molecular Biology, Central University of Jammu, J\&K, India.

Email: audeshs2002@gmail.com

Dr. Audesh Bhat

Assistant Professor and Principal Investigator, Indian Council of Medical Research-Centre for Advance Research, Shri Mata Vaishno Devi University -Katra J\&K, India, Email: kumar.rakesh@smvdu.ac.in

Dr. Rakesh Kumar

\section{References}

1. Rasool MT, et al. Cancer in Kashmir, India: burden and pattern of disease. J Cancer Res Ther. 2012;8(2):243-6.

2. Bray F, et al. Global cancer statistics 2018: GLOBOCAN estimates of incidence and mortality worldwide for 36 cancers in 185 countries. CA Cancer J Clin. 2018;68(6):394-424. 
3. al. KUNe, Survey of Patients with Cancer in Jammu and Kashmir: Based on Hospital Registry Records. Indian Journal of Computer Science and Engineering (IJCSE), 2017.

4. Bhat GR, et al. Association of newly identified genetic variant rs2853677 of TERT with non-small cell lung cancer and leukemia in population of Jammu and Kashmir, India. BMC Cancer. 2019;19(1):493.

5. Bhat A, et al. Association of ARID5B and IKZF1 variants with leukemia from Northern India. Genetic testing molecular biomarkers. 2019;23(3):176-9.

6. Verma S, et al. Genetic variants of DNAH11 and LRFN2 genes and their association with ovarian and breast cancer. Int J Gynaecol Obstet. 2020;148(1):118.

7. Shah R, et al. MassARRAY analysis of twelve cancer related SNPs in esophageal squamous cell carcinoma in J\&K, India. BMC Cancer. 2020;20(1):1-6.

8. Hecht SS. Tobacco smoke carcinogens and lung cancer. JNCl: Journal of the National Cancer Institute. 1999;91(14):1194-210.

9. Lubin $\mathrm{JH}$, et al. Lung cancer in radon-exposed miners and estimation of risk from indoor exposure. JNCl: Journal of the National Cancer Institute. 1995;87(11):817-27.

10. Bach PB, et al. Variations in lung cancer risk among smokers. J Natl Cancer Inst. 2003;95(6):470-8.

11. Ronen A, Glickman BW. Human DNA repair genes. Environ Mol Mutagen. 2001;37(3):241-83.

12. Lodish H, Zipursky BA SL, et al, DNA Damage and Repair and Their Role in Carcinogenesis. Molecular Cell Biology. 4th edition. New York: W. H. Freeman; 2000. Section 12.4.

13. lyer RR, et al. DNA mismatch repair: functions and mechanisms. Chem Rev. 2006;106(2):302-23.

14. Sale JE. Translesion DNA synthesis and mutagenesis in eukaryotes. Cold Spring Harb Perspect Biol. 2013;5(3):a012708.

15. Lange SS, Takata K-i, Wood RD. DNA polymerases and cancer. Nature reviews cancer. 2011;11(2):96-110.

16. Goričar K, et al. Translesion polymerase genes polymorphisms and haplotypes influence survival of osteosarcoma patients. Omics: a journal of integrative biology. 2015;19(3):180-5.

17. Waters LS, et al. Eukaryotic translesion polymerases and their roles and regulation in DNA damage tolerance. Microbiol Mol Biol Rev. 2009;73(1):134-54.

18. Bossé Y, Amos $\mathrm{Cl}$, A decade of GWAS results in lung cancer. 2018, AACR.

19. Yokota J, Shiraishi K, Kohno T, Genetic basis for susceptibility to lung cancer: Recent progress and future directions, in Advances in cancer research. 2010, Elsevier. p. 51-72.

20. Bhat $A$, et al. Rev3, the catalytic subunit of Polろ, is required for maintaining fragile site stability in human cells. Nucleic acids research. 2013;41(4):2328-39.

21. Zhang S, et al. REV3L 3' UTR $460 \mathrm{~T}>\mathrm{C}$ polymorphism in microRNA target sites contributes to lung cancer susceptibility. Oncogene. 2013;32(2):242.

22. Jiraskova $\mathrm{K}$, et al. Functional polymorphisms in DNA repair genes are associated with sporadic colorectal cancer susceptibility and clinical outcome. Int J Mol Sci. 2019;20(1):97. 
23. Pan J, et al. Genetic polymorphisms in translesion synthesis genes are associated with colorectal cancer risk and metastasis in Han Chinese. Gene. 2012;504(2):151-5.

24. Arnold M, et al. SNiPA: an interactive, genetic variant-centered annotation browser. Bioinformatics. 2015;31(8):1334-6.

25. Szklarczyk D, et al., The STRING database in 2017: quality-controlled protein-protein association networks, made broadly accessible. Nucleic acids research, 2016: p. gkw937.

26. Gan GN, Human REV3L: Expression and Protein Interaction Studies. 2007, University of Pittsburgh.

27. Arivazhagan R, et al., MicroRNA-340 inhibits the proliferation and promotes the apoptosis of colon cancer cells by modulating REV3L. Oncotarget, 2018. 9(4): p. 5155.

28. Varadi $\mathrm{V}$, et al. Genetic variation in genes encoding for polymerase $\zeta$ subunits associates with breast cancer risk, tumour characteristics and survival. Breast cancer research treatment. 2011;129(1):23545.

\section{Figures}

rs1002481_A allele

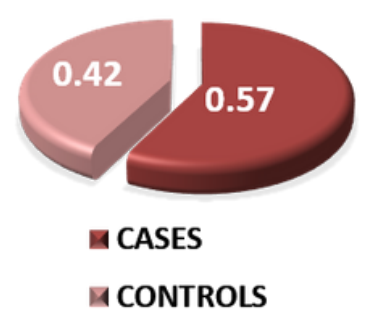

rs462779_A allele

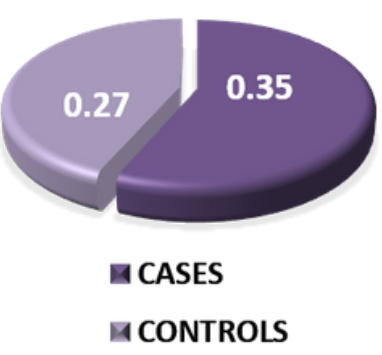

rs465646_A allele

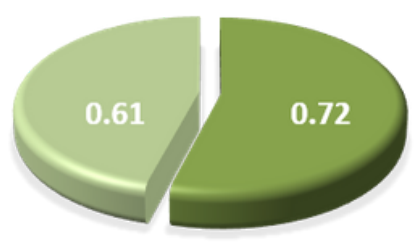

四 Cases

a Controls 
Figure 1

Risk Allele frequency in the populations of Jammu and Kashmir (Datawrapper).

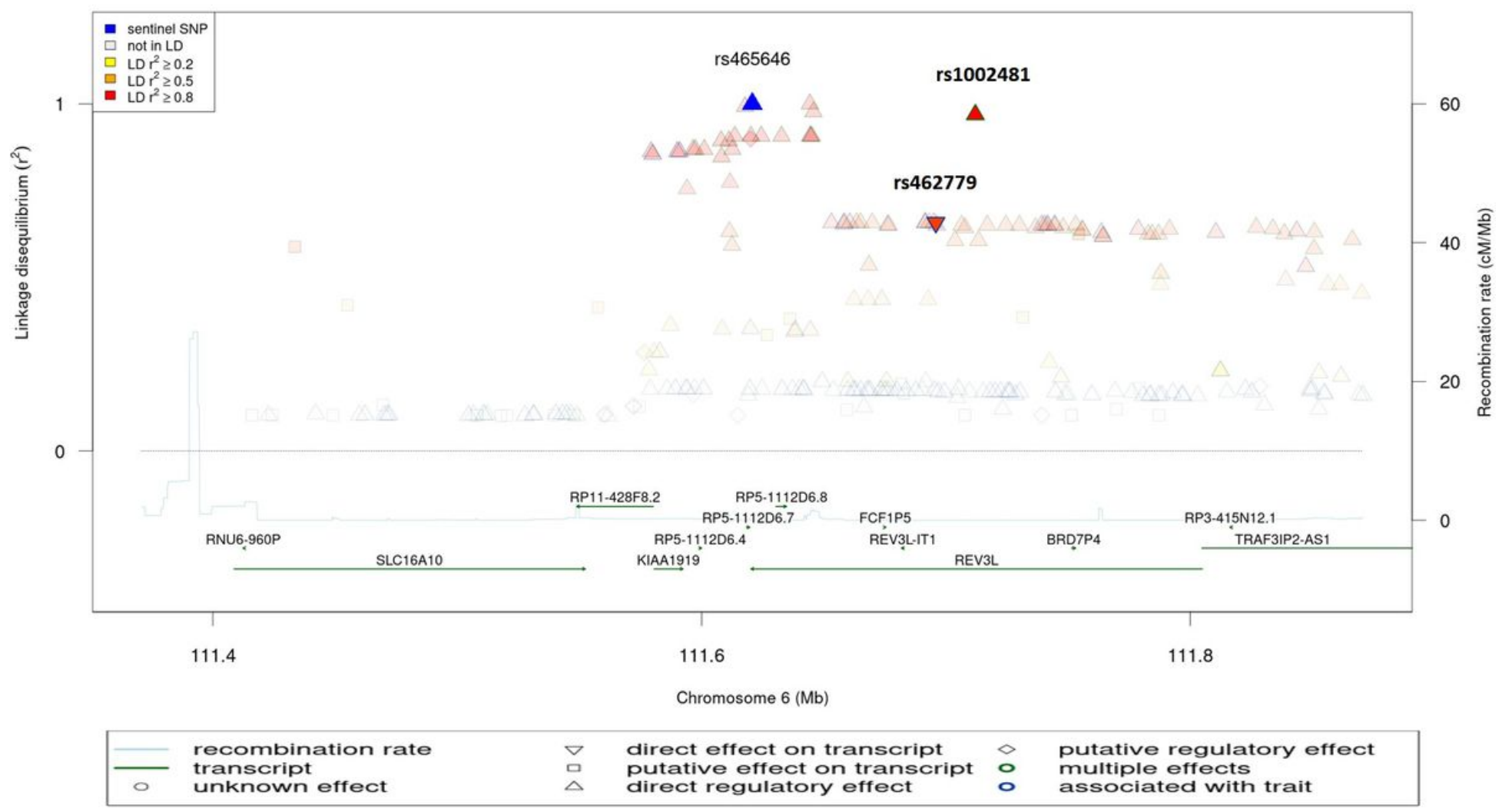

\section{Figure 2}

Linkage disequilibrium plot and the plot symbol of each variant indicating their functional annotation[24]. 


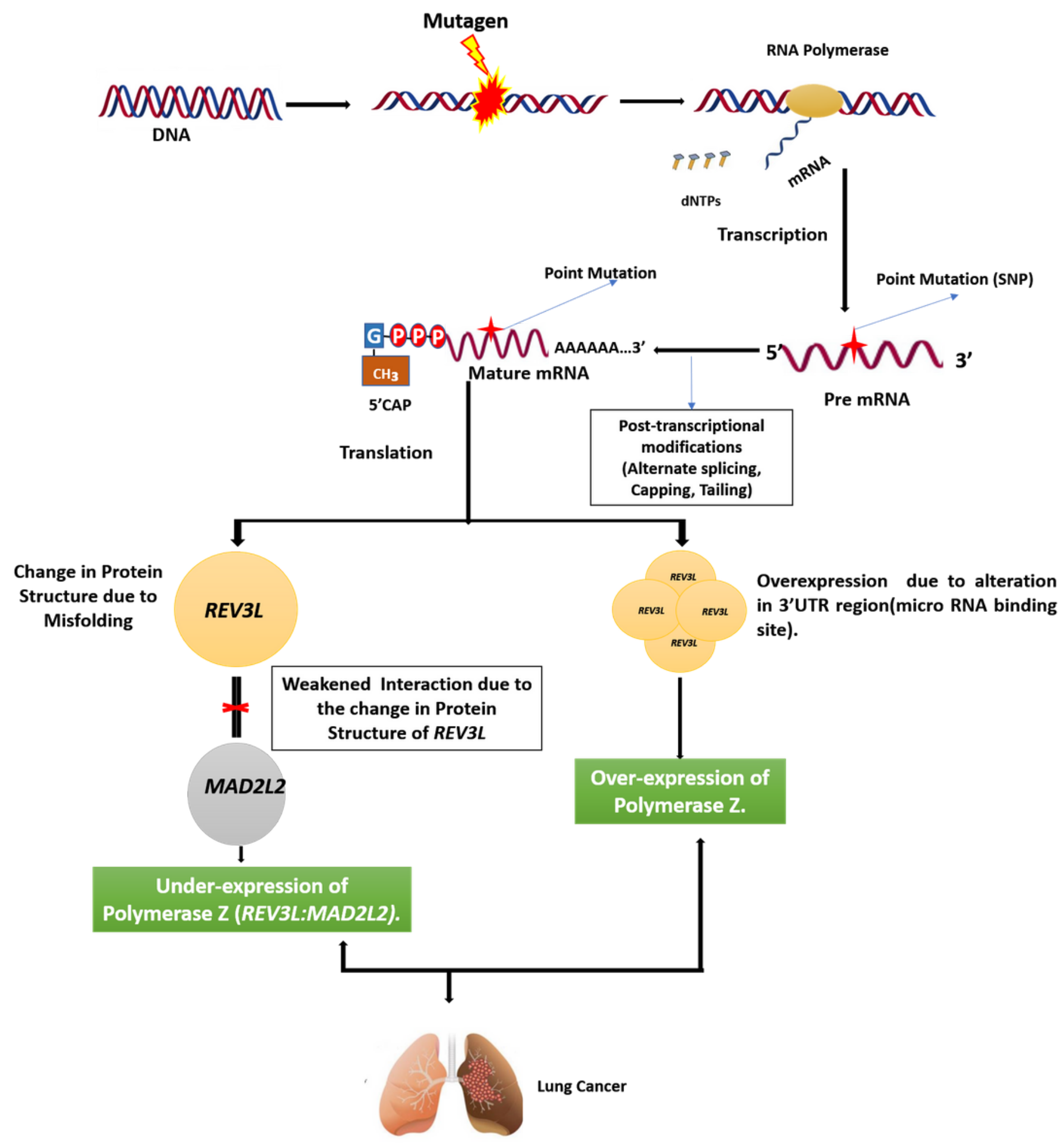

Figure 3

Schematic representation of molecular alteration in REV3L leading to both over-expression and underexpression of DNA polymerases Zeta that can increase the rate of mutation thus, promoting carcinogenesis[27].

\section{Supplementary Files}


This is a list of supplementary files associated with this preprint. Click to download.

- SupplementaryFigures.docx 